\title{
CD147 Promotes CXCL1 Expression and Modulates Liver Fibrogenesis
}

\author{
Wen-Pu Shi ${ }^{1}$, Di Ju ${ }^{2}$, Hao Li ${ }^{1}$, Lin Yuan ${ }^{3}$, Jian Cui ${ }^{1}$, Dan Luo ${ }^{1}$, Zhi-Nan Chen ${ }^{1}$ and \\ Huijie Bian ${ }^{1, *}$ \\ 1 Department of Cell Biology, National Translational Science Center for Molecular Medicine, \\ State Key Laboratory of Cancer Biology, Fourth Military Medical University, Xi'an 710032, China; \\ shiwenpu126@163.com (W.-P.S.); lhcerc@fmmu.edu.cn (H.L.); cuij93@163.com (J.C.); \\ luodan997@163.com (D.L.); zhinanchen@fmmu.edu.cn (Z.-N.C.) \\ 2 Department of Physiology, Basic Medical College, Shaanxi University of Chinese Medicine, \\ Xianyang 712046, China; judi5211314@163.com \\ 3 Clinical Laboratory, No. 457 Hospital of PLA, Wuhan 430000, China; yuanlin1987gnu@163.com \\ * Correspondence: hjbian@fmmu.edu.cn; Tel.: +86-29-84-773-243; Fax: +86-29-83-293-906
}

Received: 27 January 2018; Accepted: 3 April 2018; Published: 10 April 2018

\begin{abstract}
Activated hepatic stellate cells (HSCs) release pro-inflammatory and pro-fibrogenic factors. CXC chemokine-ligand-1 (CXCL1) is expressed on HSCs. We previously found that the CD147 is overexpressed in activated HSCs. In this study, we showed an important role of CD147 in promoting liver fibrosis by activating HSCs and upregulating expression of chemokines. Specifically, we found that CD147 specific deletion in HSCs mice alleviated $\mathrm{CCl}_{4}$-induced liver fibrosis and inhibited HSCs activation. Overexpression of CD147 upregulated the secretion of CXCL1. Meanwhile, CXCL1 promoted HSCs activation through autocrine. Treating with PI3K/AKT inhibitor could effectively suppress CD147-induced CXCL1 expression. Taken together, these findings suggest that CD147 regulates CXCL1 release in HSCs by PI3K/AKT signaling. Inhibition of CD147 attenuates $\mathrm{CCl}_{4}$-induced liver fibrosis and inflammation. Therefore, administration of targeting CD147 could be a promising therapeutic strategy in liver fibrosis.
\end{abstract}

Keywords: CD147; CXCL1; hepatic stellate cells; fibrosis

\section{Introduction}

Liver fibrosis can be caused by hepatitis B virus or hepatitis C virus infection, alcohol, non-alcoholic fatty liver disease/non-alcoholic steatohepatitis, and other relatively rare conditions, such as autoimmune hepatitis, Wilson's disease, and primary/secondary biliary cholangitis. Sustained chronic liver injury leads to normal cellular functional disruption and imbalance of the degradation and synthesis of extracellular matrix (ECM), which eventually develops the liver fibrosis [1]. Hepatic stellate cells (HSCs) exist in the space between parenchymal cells and liver sinusoidal endothelial cells of the hepatic lobule. Following chronic liver injury, HSCs activate into $\alpha-\mathrm{SMA}^{+}$contractile myofibroblast-like cells, which are characterized by the increased proliferation and migration, the unbalanced matrix degradation and the release of pro-inflammatory and pro-fibrogenic factors [2].

HSCs also have immunological functions-they express functional chemokine receptors and chemokines, including CXCL1, CXCL8, CXCL9, CXCL10, CCL2, CCL3 and CCL5 [3]. CXC chemokine-ligand-1 (CXCL1), also known as Gro-alpha, is a ligand for G-protein-coupled receptor CXCR2. CXCL1 is considered to be involved in the activation of HSCs [4], fibrogenesis and angiogenesis [5,6]. CXCL1 has also been recognized as a biomarker associated with hepatocellular carcinoma in a serum level [7].

CD147 is a transmembrane glycoprotein and a member of immunoglobulin superfamily. CD147 is widely expressed on numerous cells in carcinoma leading to the invasion, proliferation and survival of 
cancer cells, which is an important marker of tumor and poor prognosis [8-10]. Our previous studies showed that transforming growth factor- $\beta 1-C D 147$ positive feedback loop promoted the activation of HSCs, and the expression of CD147 in liver fibrosis and cirrhosis was increased which was positively correlated with the Child-Pugh grade [11,12].

In this study, we show that CD147 activates HSCs and upregulates CXCL1 expression in HSCs. In line with this, HSCs-specific CD147-knockout mice have greatly reduced HSCs activation and CXCL1 expression, leading to attenuated liver fibrosis. CD147 regulates CXCL1 expression in HSCs via PI3K/AKT pathway.

\section{Results}

\subsection{CXCL1 Expression Was Increased in Activated HSCs}

It was reported that CXCL1 expression is upregulated in $\mathrm{CCl}_{4}$-induced liver injury [5]. We examined CXCL1 level in liver tissues from normal controls and $\mathrm{CCl}_{4}$-induced mice. The CXCL1 expression showed a time-dependent increase in liver fibrotic tissues (Figure 1A). CXCL1 can be produced by liver cells, like hepatocytes and HSCs. Immunohistochemistry and immunofluorescence revealed that CXCL1 expressed in both hepatocytes and non-parenchymal liver cells (Figure 1B,C). Furthermore, an increase of CXCL1 cytoplasm expression was observed in the activated HSCs that were positive for $\alpha$-SMA in $\mathrm{CCl}_{4}$-induced mouse liver tissues (Figure 1C).

A

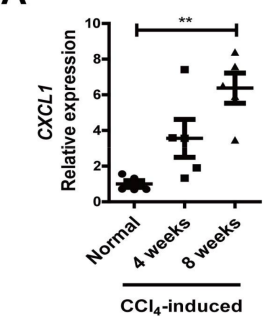

C

B
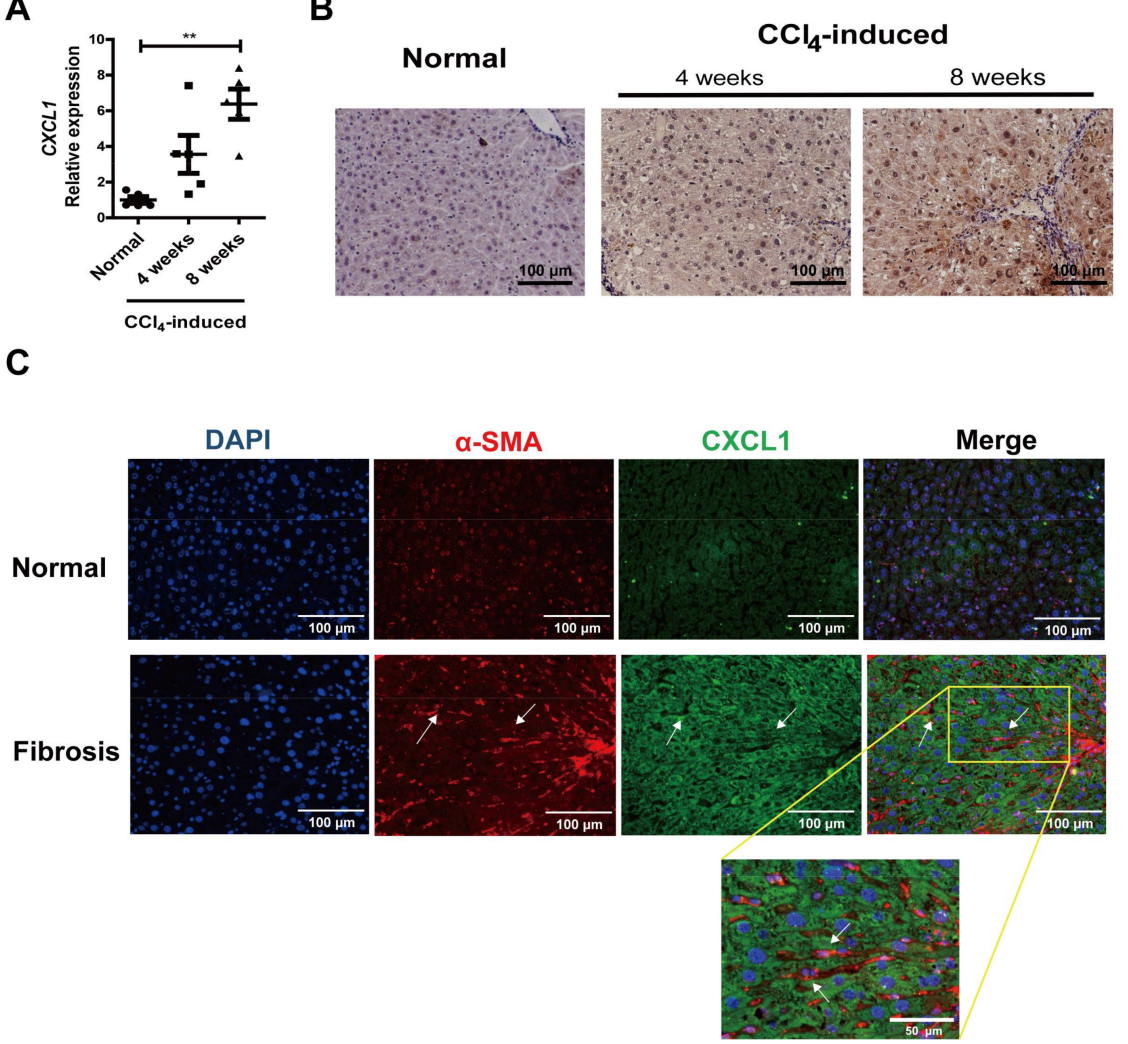

Figure 1. CXCL1 expression increased in liver fibrosis. (A) Real-time RT-PCR detection of hepatic gene expression of CXCL1 in normal control and carbon tetrachloride $\left(\mathrm{CCl}_{4}\right)$-treated mice $(n=5)$. Glyceraldehyde-3-phosphate dehydrogenase $(G A P D H)$ was used as the normalization control, ** $p<0.01$; (B) Immunohistochemistry analysis of CXCL1 in liver tissues from $\mathrm{CCl}_{4}$-induced mice; (C) Immunofluorescence detection of CXCL1 and $\alpha$-SMA in liver tissues from normal control and $\mathrm{CCl}_{4}$-induced mice (eight weeks). Arrows indicate CXCL1 (green) expression in the activated hepatic stellate cells (HSCs), which are positive for $\alpha$-SMA (red). 


\subsection{CXCL1 Promoted HSCs Activation and Co-Localized with CD147 in HSCs}

To evaluate the effect of CXCL1 on HSCs activation, LX-2 cells were treated with human recombinant CXCL1 (rCXCL1) for $24 \mathrm{~h}$ and subjected to detection of $\alpha$-SMA and type I collagen expression. Fluorescence activated cell sorting (FACS) and RT-PCR analysis showed that the expressions of $\alpha$-SMA and $\alpha 1$ (I) collagen were increased with rCXCL1 stimulation (Figure 2A,B). Cell contraction assay demonstrated that the surface area of gel was decreased (Figure 2C), indicating the cell intensive contraction after rCXCL1 treatment. The proliferation of LX-2 cells was also promoted as measured with CCK-8 assay (Figure 2D). Meanwhile, the activated HSCs showed both higher CD147 and CXCL1 expression (Figure 2E). Taken together, these results indicate that rCXCL1 promotes the activation phenotypes of HSCs.

A

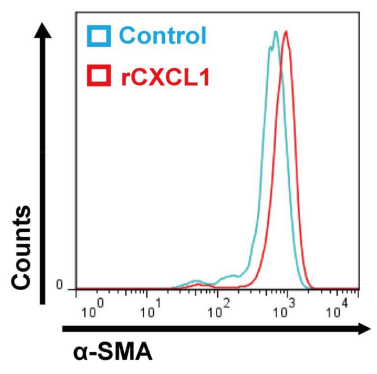

C

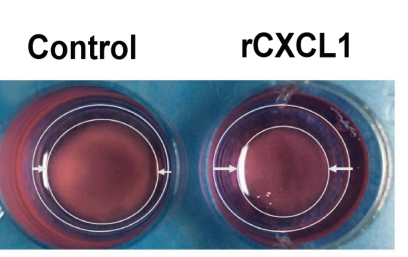

E

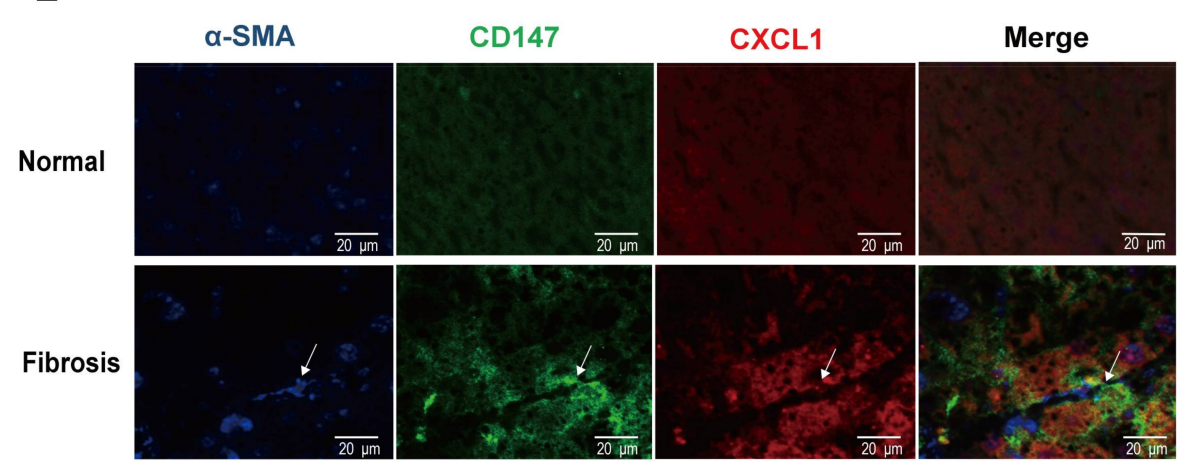

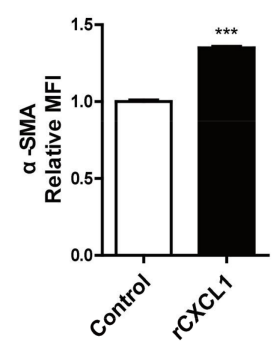

D

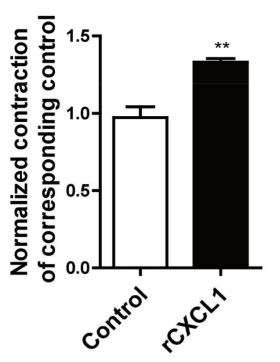

B
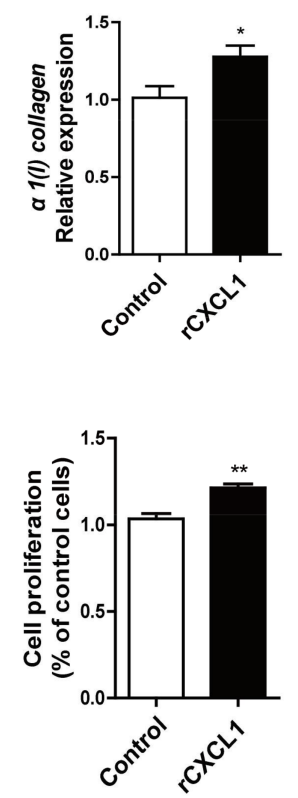

Figure 2. CXCL1 promoted HSCs activation and co-localized with CD147 in HSCs. (A) Flow cytometry analyses of $\alpha$-SMA expression; (B) Real-time RT-PCR detection of $\alpha 1$ (I) collagen mRNA level. GAPDH was used as the normalization control; (C) Representative phase contrast images and quantitative analysis of collagen-based cell contraction; (D) CCK-8 assay; (E) Immunofluorescence detection of CD147, CXCL1 and $\alpha$-SMA in liver tissues from normal control and $\mathrm{CCl}_{4}$-induced mice (eight weeks, $n=5$ ). Arrows indicate $\alpha$-SMA (blue), CD147 (green), and CXCL1 (red) expression in the activated HSCs. LX-2 cells were treated with $100 \mathrm{ng} / \mathrm{mL} \mathrm{rCXCL1} \mathrm{for} 24 \mathrm{~h}$. The results were shown as the mean \pm SD. ${ }^{*} p<0.05,{ }^{* *} p<0.01,{ }^{* * *} p<0.001$. 


\subsection{Generation of HSCs-Specific CD147-Knockout Mice}

We hypothesize that CD147 regulates the CXCL1 expression in HSCs. To obtain HSCs-specific CD147-knockout mice, we crossed the conditional CD147 targeting mice $\left(B s g^{f l / f l}\right)$ [13] with the GFAP-Cre transgenic mice. Four types of transgenic mice Bsg $f l /+, B s g^{f l / f l}$, GFAP-Cre;Bsgfl/fl and GFAP-Cre;Bsg $g^{f l /+}$ were generated (Figure 3A). The GFAP-Cre;Bsg fl/fl and $B s g f l f l$ mice were used for the following experiments. Histological analysis revealed that GFAP-Cre;Bsgflffl mice showed no spontaneous lesions in lung, heart, kidney, spleen, testis, liver and brain (Figure 3B). It was reported that GFAP mainly expresses on astrocytes in the central nervous system, while also expressing in the cartilage cells, fibroblast, hepatic epithelial cells and HSCs [14-16]. The GFAP-Cre;Bsgflffl mice showed the lower expression of CD147 in brain and liver both in the mRNA and protein levels, while there was no such significant change in other tissues (Figure 3C,D). The primary HSCs were then isolated to further verify the specific knockout of CD147 in mouse HSCs. Western blot and RT-PCR analysis showed that the expression of CD147 in isolated HSCs from GFAP-Cre;Bsg fl/fl mice was significantly reduced compared with that of Bsg fl/fl mice (Figure 3E).

\section{A}
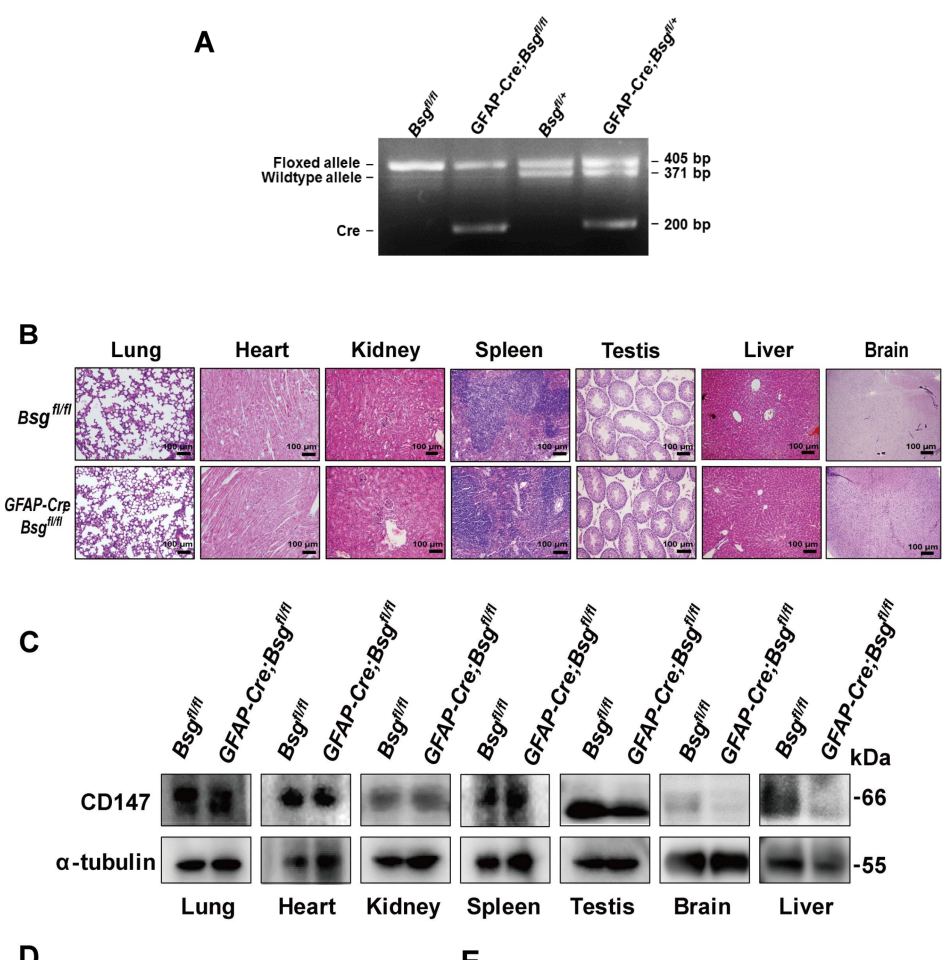

D

E
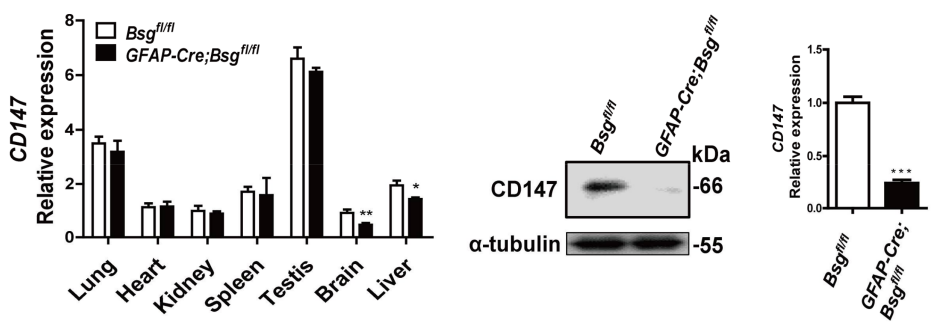

Figure 3. Generation of HSCs-specific CD147-knockout mice. (A) Identification of specific knockout of CD147 gene in the mouse genome; (B) HE stain of different tissues in Bsgflffl and GFAP-Cre;Bs gflffl; (C) Western blot; and (D) real-time RT-PCR analysis of CD147 in different tissues; (E) Western blot and real-time RT-PCR analysis of CD147 expression in mouse primary HSCs. GAPDH was used as the normalization control. The results were shown as the mean \pm SD. $n=3 .{ }^{*} p<0.05,{ }^{* *} p<0.01$, $* * * * 0.001$. 


\subsection{CD147 Deletion in HSCs Alleviated $\mathrm{CCl}_{4}$-Induced Liver Fibrosis and Deregulated CXCL1 Expression}

The GFAP-Cre;Bsgflfll and $B s g f l / f l$ mice were subjected to $\mathrm{CCl}_{4}$ intraperitoneal injection for induction of liver fibrosis. According to the anatomical structure, the mouse liver was divided into the papillary lobe, caudate lobe, right lobe, left lobe (up), left lobe (down), right middle lobe, and left middle lobe (Figure $4 \mathrm{~A}$ ). The histological images showed that $B s g f l f l$ mice had obvious pseudolobule and infiltration of inflammatory cells, whereas GFAP-Cre;Bsgflffl mice showed attenuated pseudolobule coupled with the reduced infiltration of inflammatory cells and liver damage (Figure 4B). The collagen was stained with sirius red, and the expression intensity and the percentage of positive expression area were statistically analyzed. As shown in Figure 4C, GFAP-Cre;Bsgflffl mice demonstrated the decreased collagen synthesis compared with that of $B s g f l f l l$ mice. These results suggest that the knockout of CD147 in HSCs inhibits collagen deposition and liver injury, alleviating the development of fibrosis.

We used the immunohistochemistry to detect the activated HSCs marker, as shown in Figure 4D, histological assessment showed a significant reduction of $\alpha$-SMA and desmin expression in GFAP-Cre;Bsgflfll mice. Meanwhile, the hepatic $\alpha$-SMA, desmin, and $\alpha 1$ (I) collagen gene expression were also inhibited (Figure 4E). These results suggest that HSCs' CD147 specific knockout inhibits HSCs activation during $\mathrm{CCl}_{4}$-induced fibrosis.

Then we examined CXCL1 level in primary mice HSCs without $\mathrm{CCl}_{4}$ induced by FACS. The relative median fluorescence intensity (MFI) of CXCL1 in primary GFAP-Cre;Bsgflfl mouse HSCs was reduced (Figure 5A). In addition, serum CXCL1 level was decreased in $\mathrm{CCl}_{4}$-induced GFAP-Cre;Bsg flflf mice as detected by enzyme-linked immunosorbent assay(ELISA) (Figure 5B). 
A

B
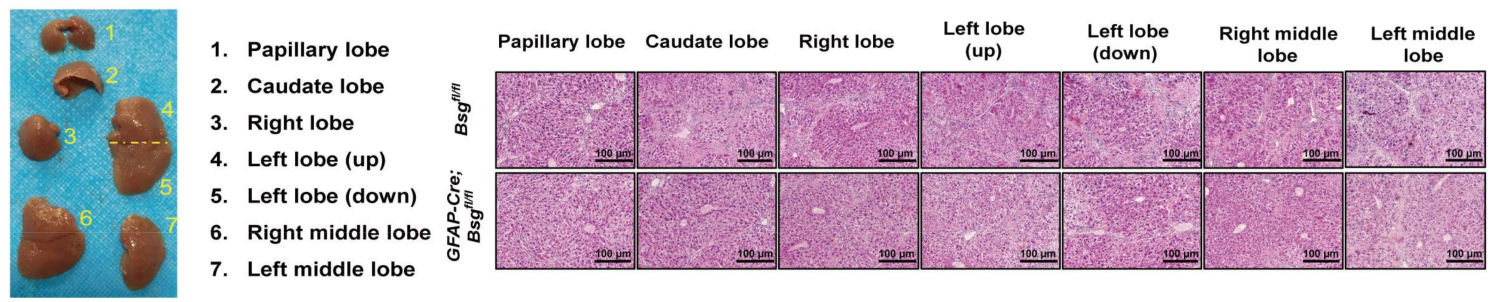

C
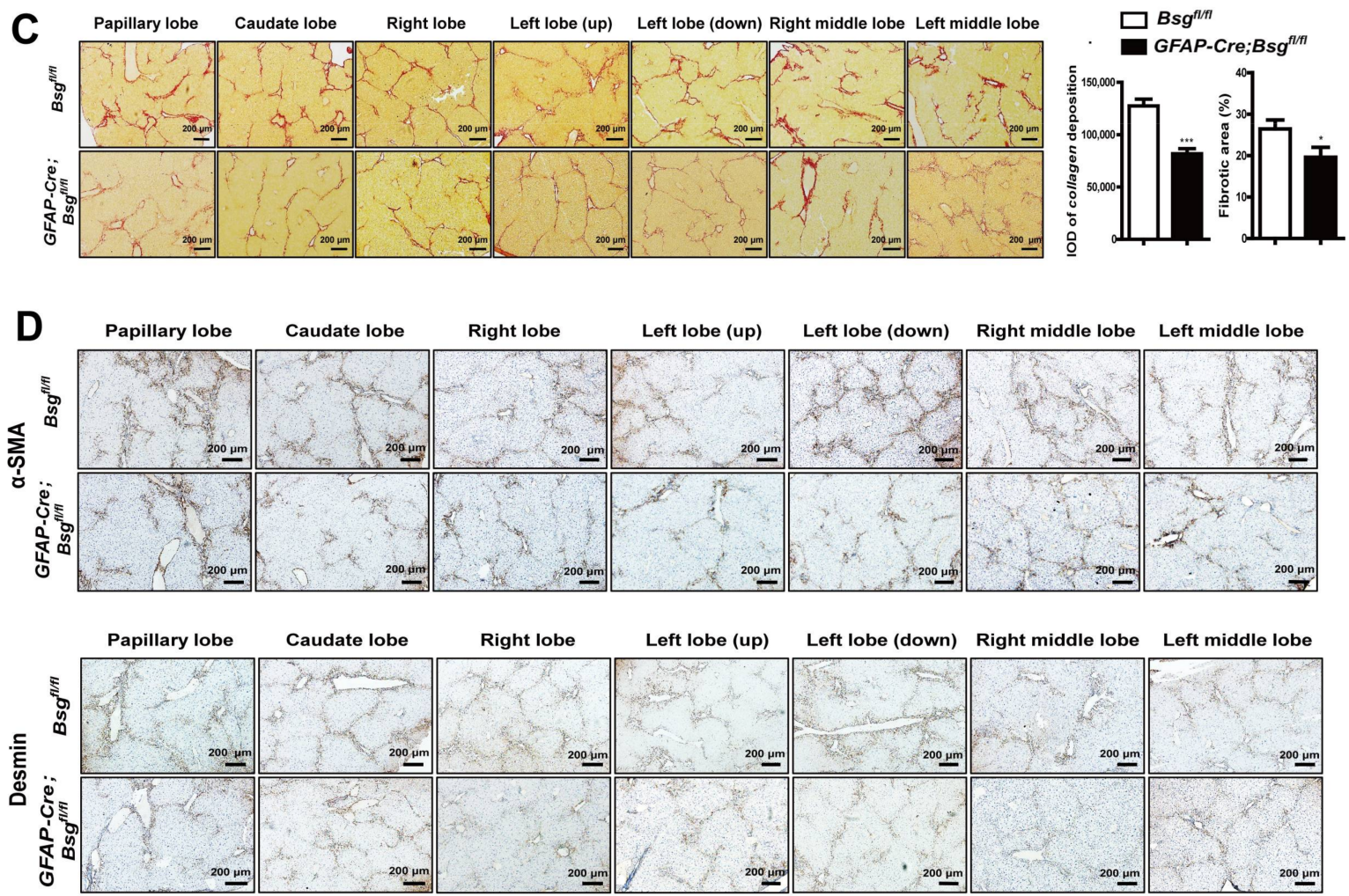

$\square B s g^{n / m}$

- GFAP-Cre;Bsg ${ }^{\text {tht }}$

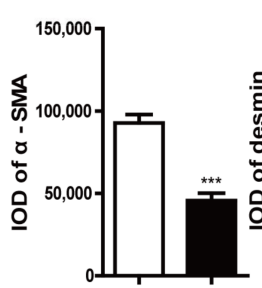

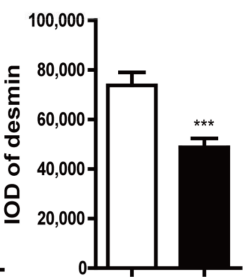

$\mathbf{E}$

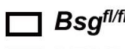

GFAP-Cre; Bsg $^{f l / f l}$

Figure 4. HSCs-specific deletion of CD147 alleviated $\mathrm{CCl}_{4}$-induced liver fibrosis $B s g f l f l$ and GFAP-Cre; $B s g f l f l$ mice treated with $\mathrm{CCl}_{4}$ for eight weeks $(n=4)$. (A) Representative photograph of seven parts of the mouse liver according to the anatomical structure; (B) Representative microphotograph of HE-stained paraffin-embedded sections of liver tissues; (C) Representative microphotograph of sirius red-stained paraffin-embedded sections of liver tissues (left), statistical analysis of integrated option density (IOD) and expression area percentage of sirius red collagen deposition (right); (D) Representative microphotograph of immunohistochemistry analysis of $\alpha$-SMA and desmin (up), statistical analysis of IOD of $\alpha$-SMA and desmin (down); (E) Real-time RT-PCR detection of hepatic gene expression of $\alpha-S M A$, desmin and $\alpha 1($ I) collagen. GAPDH was used as the normalization control. The results were shown as the mean \pm SD. ${ }^{*} p<0.05,{ }^{* * *} p<0.001$. 
A
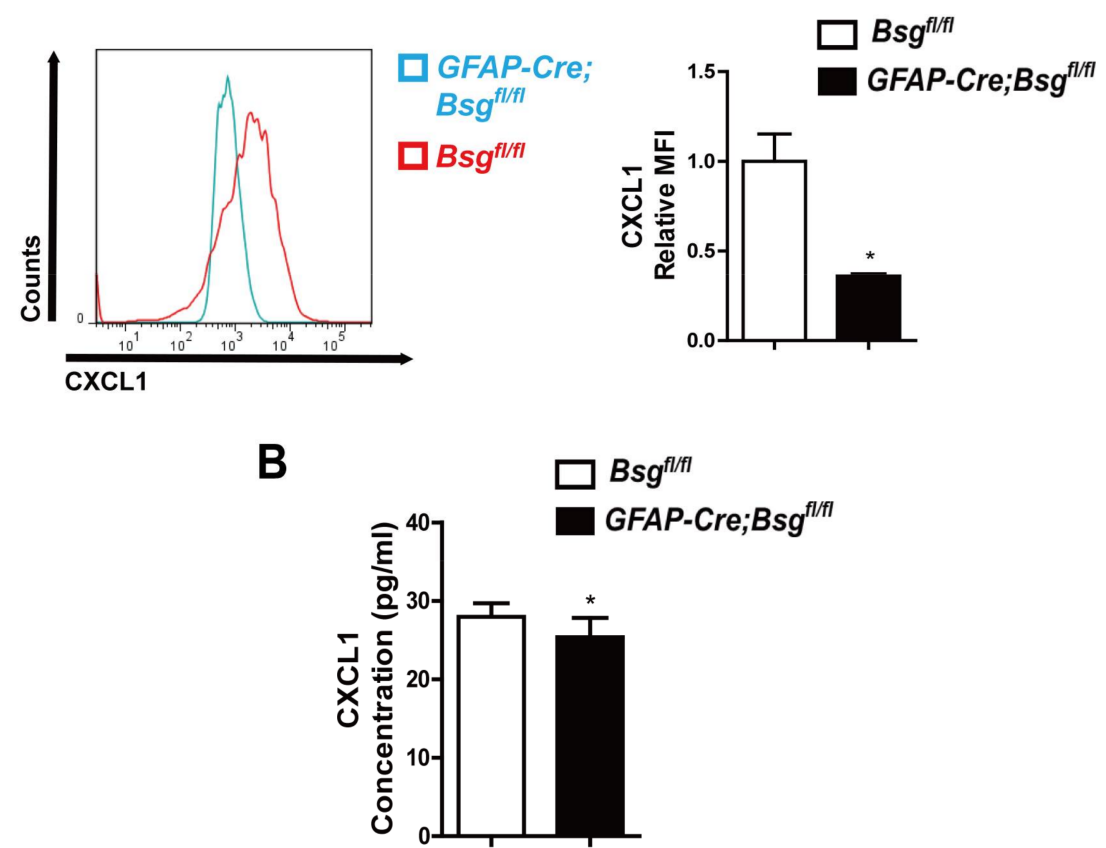

Figure 5. HSCs-specific deletion of CD147 deregulated CXCL1 expression. (A) Fluorescence activated cell sorting (FACS) analysis of CXCL1 expression in primary HSCs isolated from Bsgflffl and GFAP-Cre; Bsgflfl mice $(n=3)$; (B) Enzyme-linked immunosorbent assay (ELISA) detection of serum CXCL1 in $\mathrm{CCl}_{4}$-induced $B s g f l f l$ and GFAP-Cre; $B s g f l f l$ mice (eight weeks, $\left.n=4\right)$. The results were shown as the mean \pm SD. ${ }^{*} p<0.05$.

\subsection{CD147-Regulated CXCL1 Expression in HSCs via the PI3K/AKT Pathway}

Transient over-expression of CD147 increased the CXCL1 expression in LX-2 cells (Figure 6A). Next, we evaluated the effects of silencing CD147 in LX-2 cells by siRNA. Knockdown of CD147 by si-CD147 decreased the expression of CXCL1 compared with that of silencer-negative control siRNA (snc-RNA) (Figure 6B). These results demonstrate that CD147 promotes CXCL1 expression in HSCs, which is consistent with the previous hypothesis. To investigate whether CD147 regulated CXCL1 through PI3K/AKT pathway, we treated LX-2 cells with $10 \mu \mathrm{mol} / \mathrm{L}$ LY294002, a PI3K inhibitor. The results showed that CD147 overexpression induced the AKT phosphorylation (p-AKT) and elevated CXCL1 expression. Meanwhile, the CD147-induced CXCL1 expression was significantly inhibited by the selective FAK/PI3K inhibitor LY294002 (Figure 6C). All of these results indicated the crucial role of activated PI3K/AKT signaling in CD147-regulated CXCL1 expression. 
A
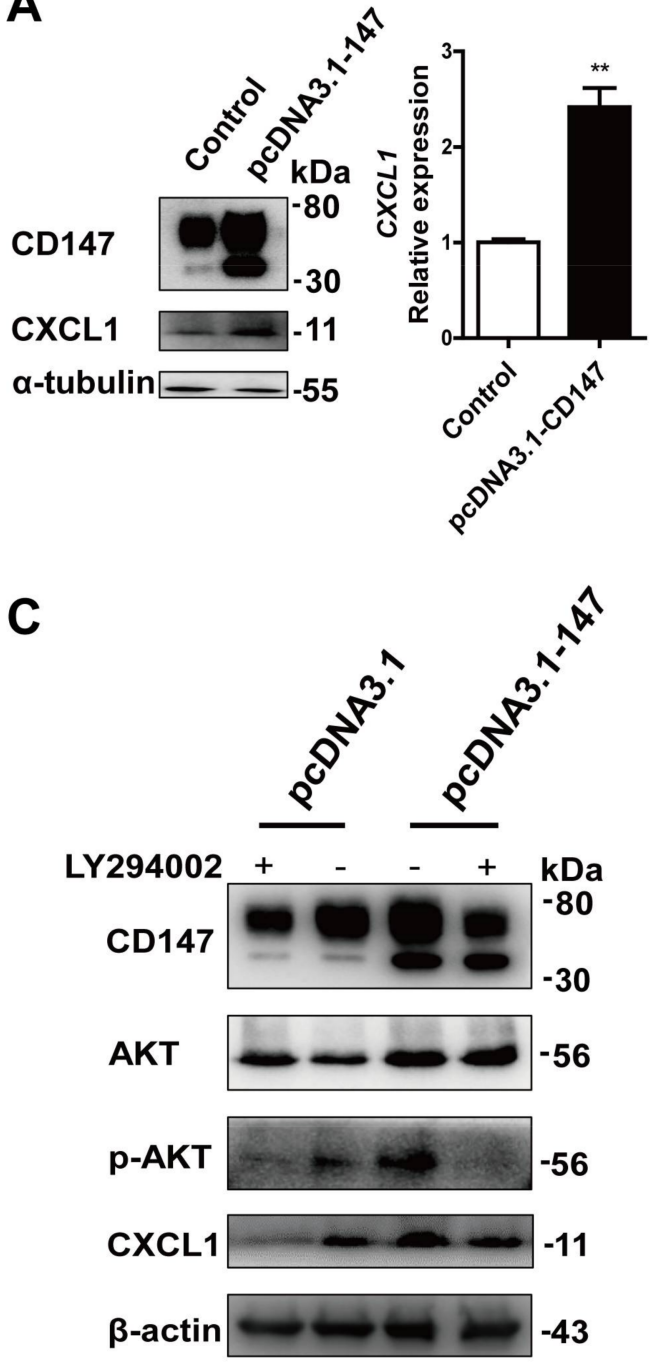

B
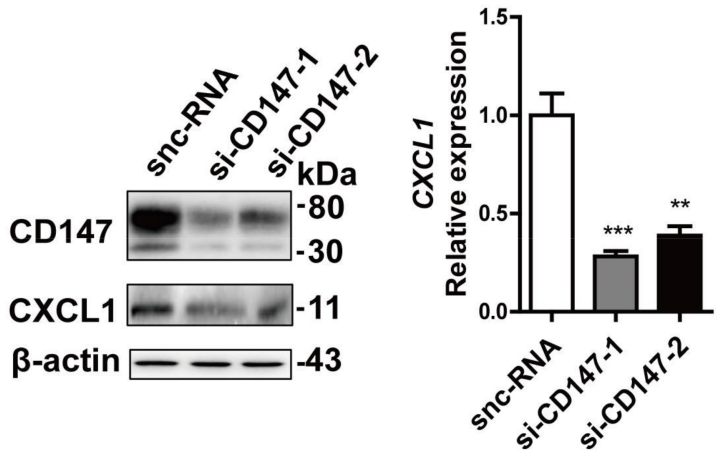

Figure 6. CD147 regulated CXCL1 expression in HSCs via PI3K/AKT pathway. (A) Western blot and real-time RT-PCR analysis of CD147 and CXCL1 in LX-2 cells that transfected with pcDNA3.1-CD147; (B) Western blot and real-time RT-PCR analysis of CD147 in LX-2 cells transfected with CD147 siRNA. LX-2 cells were transfected with sncRNA as the control; (C) Western blot analysis of CD147, AKT, p-AKT, and CXCL1 in pcDNA3.1-CD147-transfected LX-2 cells that were pre-incubated with $10 \mu \mathrm{mol} / \mathrm{L}$ LY294002. Western densitometry was performed for three independent experiments. Cells were transfected with pcDNA3.1(+) as the control. GAPDH was used as the normalization control. The results were shown as the mean $\pm \mathrm{SD} .{ }^{*} p<0.05,{ }^{* *} p<0.01,{ }^{* * *} p<0.001$.

\section{Discussion}

Patients with fibrosis often result from different etiologies of chronic liver injury. Cirrhosis is the terminal stage of progressive liver fibrosis, eventually developing to the hepatocellular carcinoma. Liver fibrosis is shown to be a reversible process [17-19], withdrawal of the chronic injury results in decrease of pro-inflammatory and fibrogenic cytokines, increased collagenase activity, decreased ECM production, and disappearance of hepatic-activated HSCs [20-22]. Our previous results show that CD147 upregulates fibrosis-related marker $\alpha$-SMA and $\alpha 1$ (I) collagen in HSCs, and the positive loop of transforming growth factor- $\beta 1-C D 147$ promoted the activation of HSCs [11,12]. Here, we provided additional evidence that knockout CD147 in mouse HSCs inhibited collagen deposition, inflammatory infiltration and $\mathrm{HSC}$ activation, alleviating $\mathrm{CCl}_{4}$-induced liver fibrosis. In addition, we found that overexpression of CD147 in LX-2 cells upregulated expression of chemokine CXCL1, which 
was upregulated in activated HSCs. Moreover, CXCL1 exerted a positive activation loop on HSCs, enhancing HSCs activation phenotypes. Taken together, these findings support our conclusions that CD147 stimulates the release of chemokine CXCL1 and promotes the development and progression of liver fibrosis.

Accumulated evidence suggests that chemokines play a critical role in acute and chronic liver diseases, mediating the immune infiltration in injured liver. Additionally, chemokines can also directly affect the biology of liver resident cells, such as HSCs, during inflammatory and fibrogenic tissue responses [23]. It is reported the increased hepatic expression of pro-inflammatory genes TNF, EMR1, CCL2, MPO, CXCL1 and CXCL2 in liver injury [24]. In our study, we found that CXCL1 in activated HSCs was upregulated in $\mathrm{CCl}_{4}$-induced fibrosis. CXCL1 binds to the CXCR2, which expresses on HSCs and neutrophils, and several types of cancer including melanoma [25], lung [26] and pancreatic cancers [27]. Stimulation of HSCs with recombinant CXCL1 results in increased collagen type I and $\alpha$-SMA production $[5,28]$. Similarly, in our study rCXCL1 also upregulated the activation markers, $\alpha$-SMA and $\alpha 1$ (I) collagen in LX-2 cells and promoted the cell contraction and proliferation.

We used the transgenic mouse GFAP-Cre;Bsg fl/fl to further verify the CD147 function in HSCs in our study. Liver injury is usually accompanied with inflammatory response. A high-fat diet combined with acute ethanol consumption synergistically induces acute liver inflammation and injury via upregulation of hepatic CXCL1 expression and promotion of hepatic neutrophil infiltration [29]. In $\mathrm{CCl}_{4}$-induced mouse fibrosis, GFAP-Cre;Bsgflfll mice showed less hepatic inflammatory infiltration and serum CXCL1 expression. We had proven that overexpression of CD147 promoted $\alpha 1$ (I) collagen synthesis, while GFAP-Cre;Bsgflffl mice showed less amount of collagen deposition. Liver fibrosis is due to the unbalanced degradation and synthesis of ECM. Activated HSCs are a major source of collagen and ECM and play a key role in the formation of hepatic fibrosis [30,31]; quantitative assessment of HSCs activity is one of the means to detect the development of fibrosis. The GFAP-Cre;Bsgflffl mice showed a reduction of $\alpha$-SMA and desmin expression, which indicates that inhibiting CD147 contributes to the HSCs deactivation and, thus, the reversal of HSC activation contributes to termination of fibrogenesis [32].

The activated HSCs have activation signatures (MAPK and PI3K-AKT phosphorylation) and upregulated genes (COL1A1, $\alpha$-SMA, and CXCL1) expression [33]. As integrin is important for HSCs transdifferentiation [34], in integrin-mediated signaling transduction process, FAK is a key molecule and acts like an integrator that accepts and amplifies signals in the cell and activates downstream signal pathway [35]. CD147 can bind to integrin and activates the downstream FAK/PI3K signaling pathway [36]. CD147 overexpression induced the AKT phosphorylation; however, treating with FAK/PI3K inhibitor LY294002, CD147-induced AKT phosphorylation and CXCL1 expression were significantly inhibited. Taken together, CD147 mediated CXCL1 expression probably by directly binding to integrin in HSCs through the PI3K/AKT cell signal activation.

Liver fibrosis shows complex interplay between the epithelial cells, inflammatory cells, myofibroblasts and ECM components of the wound-healing response [2]. In summary, we demonstrate that CD147 promotes the CXCL1 expression and modulates the liver fibrosis. Therapeutically, our results strongly suggest a potential for inhibition of CD147 as a treatment strategy in liver fibrosis.

\section{Materials and Methods}

\subsection{Cells Culture and Reagents}

Human HSCs cell line, LX-2 was cultured in Dulbecco's Modified Eagle Medium (DMEM) (Hycolon, Logan, UT, USA) supplemented with antibiotics $(100 \mathrm{U} / \mathrm{mL}$ of penicillin and $100 \mathrm{mg} / \mathrm{mL}$ of streptomycin) and $10 \%$ fetal bovine serum in a humidified atmosphere containing $5 \% \mathrm{CO}_{2}$ at 37 ${ }^{\circ} \mathrm{C}$. Mouse primary HSCs were isolated from the mice livers mainly by in situ pronase/collagenase perfusion of mouse liver, followed by density gradient-based separation, as described [37]. The cells were cultured in DMEM (Hycolon) supplemented with antibiotics $(100 \mathrm{U} / \mathrm{mL}$ of penicillin and 
$100 \mathrm{mg} / \mathrm{mL}$ of streptomycin) and 15\% fetal bovine serum. Human recombinant CXCL1 was from Peprotech (Princeton, NJ, USA). PI3K/AKT inhibitor, LY294002 was from Cell Signaling Technology (Danvers, MA, USA).

\subsection{Mice}

All experimental protocols were approved by the Laboratory Animal Ethics Committee of the Fourth Military Medical University (No. 20121003, 16 October 2012) and performed in strict accordance with the People's Republic of China Legislation Regarding the Use and Care of Laboratory Animals. HSCs-specific Cre recombinase transgenic mice (glial fibrillary acidic protein (GFAP)-Cre) purchased from Shanghai Biomodel Organism Science \& Technology Development Co., Ltd. (Shanghai, China) and conditional CD147 targeting mice $\left(B s g^{f l f f l}\right)$ were constructed by Dr. Yao Hui [13] in our laboratory. All gene identification primers were synthesized by the Beijing Genomics Institute (BGI, Beijing, China) and listed in Table 1 . To induce chronic liver injury, mice were treated with $10 \%$ carbon tetrachloride $\left(\mathrm{CCl}_{4}\right)$ intraperitoneally at a dose of $5 \mu \mathrm{L} / \mathrm{g}$, three times per week.

Table 1. Sequences of PCR primers.

\begin{tabular}{ccc}
\hline Gene & Forward Sequence $\left(\mathbf{5}^{\prime}-\mathbf{3}^{\prime} \mathbf{)}\right.$ & Reverse Sequence $\mathbf{( 5}^{\prime}-\mathbf{3}^{\prime} \mathbf{)}$ \\
\hline Mouse GFAP Cre & ACTCCTTCATAAAGCCCTCG & ATCACTCGTTGCATCGACCG \\
Mouse CD147 Loxp & ATAGAAATGGGGGATGCTCTG & GGCTCTGTCTTCACTTGGGTT \\
Human CXCL1 & ATGGCCCGCGCTGCTCTCTCC & GTTGGATTTGTCACTGTTCAG \\
Human CD147 & ACTCCTCACCTGCTCCTTGA & GCCTCCATGTTCAGGTTCTC \\
Human $\alpha 1(I)$ collagen & AACATGACCAAAAACCAAAAGT & CATTGTTTCCTGTGTCTTCTGG \\
Human GAPDH & GCACCGTCAAGGCTGAGAAC & ATGGTGGTGAAGACGCCAGT \\
Mouse CD147 & TGGCAAGTATGTGGTGGTAT & GTGAGATGGTTTCCCGAGT \\
Mouse $\alpha$-SMA & GTCCCAGACATCAGGGAGTAA & TCGGATACTTCAGCGTCAGGA \\
Mouse desmin & AACAGCCTCGGTTCCTTGAG & GACCTGAGGCTAAACAGGCG \\
Mouse $\alpha 1(I)$ collagen & GCTCCTCTTAGGGGCCACT & CCACGTCTCACCATTGGGG \\
Mouse CXCL1 & CACAGGGGCGCCTATCGCCAA & CAAGGCAAGCCTCGCGACCAT \\
Mouse GAPDH & AGGTCGGTGTGAACGGATTTG & TGTAGACCATGTAGTTGAGGTCA \\
\hline
\end{tabular}

\section{3. siRNA Transfection}

Silencing was performed by the transient transfection of siRNA oligos using Lipofectamine 2000 (Invitrogen, Carlsbad, CA, USA) following the manufacturer's instructions. We used two silencing oligos from GenePharma (Shanghai, China): si-CD147-1: 5'-GTA CAA GAT CAC TGA CTC T-3'; si-CD147-2: 5'-GTT CTT CGT GAG TTC CTC-3'.

\subsection{Western Blot}

Western blot was performed as previously described [11]. The primary antibodies used were mouse anti-human CD147 antibody (1:1000) prepared by our laboratory [38], anti-AKT (1:2000; ARE6004), anti-phospho-AKT (1:500; ARE6002) were purchased from Antibody revelation (San Diego, CA, USA), rat anti-mouse CD147 (1:500; ab34016) were purchased from Abcam (Cambridge, UK), anti- $\beta$-actin (1:1000; M1210-2) were purchased from Hubio (Hangzhou, China), and anti- $\alpha$-tubulin (1:1000; 66031-1-Ig) were purchased from Proteintech (Wuhan, China). A Western-Light chemiluminescent detection system (Image Station 4000 MM Pro, XLS180, Kodak, Rochester, NY, USA) was used to visualize the signals.

\subsection{Real-Time $P C R$}

First, total RNA was extracted by a Total RNA Kit II (Omega, Riverside, CA, USA), then using a PrimeScript ${ }^{\mathrm{TM}}$ RT reagent kit (TaKaRaBio, Otsu, Japan) reverse transcript to cDNA. All the steps were based on the product protocol. Single-stranded cDNA was amplified by quantitative RT-PCR using a SYBR Premix ExTaq ${ }^{\mathrm{TM}}$ kit (TaKaRaBio) on a Stratagene Mx3005P ${ }^{\mathrm{TM}}$ Real-Time PCR System (Agilent Technologies, Waldbronn, Germany). Glyceraldehyde-3-phosphate dehydrogenase (GAPDH) 
mRNA was used to normalize RNA inputs. RT reactions were performed as previously described [11]. All primers were synthesized by the Beijing Genomics Institute (BGI, Beijing, China) and listed in Table 1.

\subsection{Enzyme-Linked Immunosorbent Assay (ELISA)}

First, we used serum separator tube and allowed mice blood samples to clot for one hour at room temperature, then centrifuged for $15 \mathrm{~min}$ at approximately $3000 \times g$, at $4{ }^{\circ} \mathrm{C}$. Serum CXCL1 detection was performed using a CXCL1 enzyme-linked immunosorbent assay kit (Mlbio, Shanghai, China) according to the manufacturer's instructions. The concentration in each sample well was determined by interpolation from a standard curve. Each sample was tested in triplicate.

\subsection{Cell Contraction Assay}

We used a standard kit assay (Cell Biolabs, San Diego, CA, USA), operated according to the manufacturer's instructions. The gel area was quantified by ImageJ software (Image 1.48, National Institutes of Health). The gel contraction area was calculated by the initial gel area (outer white circle) minus the terminal gel area (inner white circle). The normalized contraction of corresponding control was a ratio of control contraction area to rCXCL1 contraction area.

\subsection{Collagen Staining}

Collagen accumulation was detected with sirius red, which was performed as previously described [37]. Liver tissues were fixed with $4 \%$ formalin and embedded in paraffin. Following deparaffinization and hydration, the sections were stained with sirius red (Sigma, Darmstadt, Germany).

\subsection{Immunohistochemistry}

Liver tissues were fixed with $4 \%$ formalin and embedded in paraffin. Sections were deparaffinized and incubated with primary antibodies including anti-CXCL1 (1:100; ab86436), anti- $\alpha$-SMA (1:100; ab7817), and anti-desmin (1:100; ab86592) (Abcam, Cambridge, UK). Staining was performed as described previously [39].

\subsection{Immunofluorescence}

Immunofluorescence was carried out as described previously [39]. Primary antibodies included anti- $\alpha$-SMA (1:100; ab7817), anti-CXCL1 (1:100; ab86436) and anti-CD147 antibodies (1:100; ab34016) (Abcam, Cambridge, UK).

\subsection{Fluorescence Activated Cell Sorting (FACS)}

FACS analysis was carried out as described previously [40]. Primary antibodies included anti- $\alpha$-SMA (1:100; ab7817) (Abcam, Cambridge, UK) and anti-CXCL1 (1:100; MAB4542) (RD, Minneapolis, MN, USA). The cells were analyzed by flow cytometry (BD FACSCalibur ${ }^{\mathrm{TM}}$ Flow Cytometer, BD Bioscience, San Jose, CA, USA) using FlowJo software (FlowJo 7.6, BD Bioscience).

\subsection{CCK-8}

LX-2 cells $\left(5 \times 10^{4}\right.$ cells/well) were seeded in 96-well plates and incubated with $100 \mathrm{ng} / \mathrm{mL}$ rCXCL1 for $24 \mathrm{~h}$. Ten $\mu \mathrm{L}$ of the CCK8 (Engreen, Beijing, China) was added to each well using the pipettor. The cells were incubated for $2 \mathrm{~h}$. The absorbance of each sample was measured using a microplate reader at a wavelength of $450 \mathrm{~nm}$. 


\subsection{Statistical Analysis}

Each experiment was repeated at least three times. Student's $t$-test was used to compare the two mean values. A one-way analysis of variance was performed to compare the multiple mean values. Data were presented as the mean \pm SD from three independent experiments unless otherwise indicated. The Graphpad Prism software and SPSS 17.0 software were used for statistical analysis. A $p$ value $<0.05$ was considered statistically significant.

\section{Conclusions}

In summary, we demonstrate that CD147 promotes the CXCL1 expression in HSCs and modulates liver fibrosis. Therapeutically, our results strongly suggest a potential for inhibition of CD147 as a treatment strategy in liver fibrosis.

Acknowledgments: We thank Scott L. Friedman (Mount Sinai School of Medicine, USA) for providing LX-2 cell line. This work was supported by a grant from the National Natural Science Foundation of China (31571434) and the National Basic Research Program of China (2015CB553701).

Author Contributions: Wen-Pu Shi, Di Ju and Huijie Bian designed research; Hao Li, Lin Yuan, Jian Cui and Dan Luo were involved in the methodology; investigation was done by Wen-Pu Shi, Di Ju; Wen-Pu Shi and Hujie Bian were involved in the writing-original draft; Huijie Bian and Zhi-Nan Chen were involved in the writing-review and editing; Huijie Bian and Zhi-Nan Chen were involved in funding acquisition; supervision was done by Zhi-Nan Chen and Huijie Bian. All authors read and approved the manuscript.

Conflicts of Interest: The authors declare no conflict of interest.

\section{Abbreviations}

$\begin{array}{ll}\alpha \text {-SMA } & \alpha \text {-smooth muscle actin } \\ \text { CXCL1 } & \text { CXC chemokine-ligand-1 } \\ \mathrm{CCl}_{4} & \text { Carbon tetrachloride } \\ \text { ECM } & \text { Extracellular matrix } \\ \text { ELISA } & \text { Enzyme-linked immunosorbent assay } \\ \text { FACS } & \text { Fluorescence activated cell sorting } \\ \text { GFAP } & \text { Glial fibrillary acidic protein } \\ \text { HSC } & \text { Hepatic stellate cell } \\ \text { MFI } & \text { Median fluorescence intensity } \\ \text { snc-RNA } & \text { Silencer-negative control siRNA }\end{array}$

\section{References}

1. Higashi, T.; Friedman, S.L.; Hoshida, Y. Hepatic stellate cells as key target in liver fibrosis. Adv. Drug Deliv. Rev. 2017, 121, 27-42. [CrossRef] [PubMed]

2. Pellicoro, A.; Ramachandran, P.; Iredale, J.P.; Fallowfield, J.A. Liver fibrosis and repair: Immune regulation of wound healing in a solid organ. Nat. Rev. Immunol. 2014, 14, 181-194. [CrossRef] [PubMed]

3. Sahin, H.; Trautwein, C.; Wasmuth, H.E. Functional role of chemokines in liver disease models. Nat. Rev. Gastroenterol. Hepatol. 2010, 7, 682-690. [CrossRef] [PubMed]

4. Nischalke, H.D.; Berger, C.; Luda, C.; Muller, T.; Berg, T.; Coenen, M.; Kramer, B.; Korner, C.; Trebicka, J.; Grunhage, F; et al. The CXCL1 rs4074 A allele is associated with enhanced CXCL1 responses to TLR2 ligands and predisposes to cirrhosis in HCV genotype 1-infected Caucasian patients. J. Hepatol. 2012, 56, 758-764. [CrossRef] [PubMed]

5. Stefanovic, L.; Brenner, D.A.; Stefanovic, B. Direct hepatotoxic effect of KC chemokine in the liver without infiltration of neutrophils. Exp. Biol. Med. 2005, 230, 573-586. [CrossRef]

6. Strieter, R.M.; Burdick, M.D.; Mestas, J.; Gomperts, B.; Keane, M.P.; Belperio, J.A. Cancer CXC chemokine networks and tumour angiogenesis. Eur. J. Cancer 2006, 42, 768-778. [CrossRef] [PubMed]

7. Wu, F.X.; Wang, Q.; Zhang, Z.M.; Huang, S.; Yuan, W.P.; Liu, J.Y.; Ban, K.C.; Zhao, Y.N. Identifying serological biomarkers of hepatocellular carcinoma using surface-enhanced laser desorption/ionization-time-of-flight mass spectroscopy. Cancer Lett. 2009, 279, 163-170. [CrossRef] [PubMed] 
8. Grass, G.D.; Dai, L.; Qin, Z.; Parsons, C.; Toole, B.P. CD147: Regulator of hyaluronan signaling in invasiveness and chemoresistance. Adv. Cancer Res. 2014, 123, 351-373. [PubMed]

9. Kanekura, T.; Chen, X. CD147/basigin promotes progression of malignant melanoma and other cancers. J. Dermatol. Sci. 2010, 57, 149-154. [CrossRef] [PubMed]

10. Partridge, E.A.; Le Roy, C.; Di Guglielmo, G.M.; Pawling, J.; Cheung, P.; Granovsky, M.; Nabi, I.R.; Wrana, J.L.; Dennis, J.W. Regulation of cytokine receptors by Golgi N-glycan processing and endocytosis. Science 2004, 306, 120-124. [CrossRef] [PubMed]

11. Li, H.Y.; Ju, D.; Zhang, D.W.; Li, H.; Kong, L.M.; Guo, Y.; Li, C.; Wang, X.L.; Chen, Z.N.; Bian, H. Activation of TGF-beta1-CD147 positive feedback loop in hepatic stellate cells promotes liver fibrosis. Sci. Rep. 2015, 5, 16552. [CrossRef]

12. Zhang, D.W.; Zhao, Y.X.; Wei, D.; Li, Y.L.; Zhang, Y.; Wu, J.; Xu, J.; Chen, C.; Tang, H.; Zhang, W.; et al. HAb18G/CD147 promotes activation of hepatic stellate cells and is a target for antibody therapy of liver fibrosis. J. Hepatol. 2012, 57, 1283-1291. [CrossRef] [PubMed]

13. Yao, H.; Teng, Y.; Sun, Q.; Xu, J.; Chen, Y.T.; Hou, N.; Cheng, X.; Yang, X.; Chen, Z.N. Important functional roles of basigin in thymocyte development and T cell activation. Int. J. Biol. Sci. 2013, 10, 43-52. [CrossRef] [PubMed]

14. Maubach, G.; Lim, M.C.; Zhang, C.Y.; Zhuo, L. GFAP promoter directs lacZ expression specifically in a rat hepatic stellate cell line. World J. Gastroenterol. 2006, 12, 723-730. [CrossRef]

15. Neubauer, K.; Knittel, T.; Aurisch, S.; Fellmer, P.; Ramadori, G. Glial fibrillary acidic protein-A cell type specific marker for Ito cells in vivo and in vitro. J. Hepatol. 1996, 24, 719-730. [CrossRef]

16. Puche, J.E.; Lee, Y.A.; Jiao, J.; Aloman, C.; Fiel, M.I.; Munoz, U.; Kraus, T.; Lee, T.; Yee, H.F., Jr.; Friedman, S.L. A novel murine model to deplete hepatic stellate cells uncovers their role in amplifying liver damage in mice. Hepatology 2013, 57, 339-350. [CrossRef] [PubMed]

17. Iimuro, Y.; Nishio, T.; Morimoto, T.; Nitta, T.; Stefanovic, B.; Choi, S.K.; Brenner, D.A.; Yamaoka, Y. Delivery of matrix metalloproteinase-1 attenuates established liver fibrosis in the rat. Gastroenterology 2003, 124, 445-458. [CrossRef] [PubMed]

18. Ramachandran, P.; Iredale, J.P. Reversibility of liver fibrosis. Ann. Hepatol. 2009, 8, 283-291. [PubMed]

19. Sun, M.; Kisseleva, T. Reversibility of liver fibrosis. Clin. Res. Hepatol. Gastroenterol. 2015, 39, S60-S63. [CrossRef] [PubMed]

20. Atta, H.M. Reversibility and heritability of liver fibrosis: Implications for research and therapy. World J. Gastroenterol. 2015, 21, 5138-5148. [CrossRef]

21. Huang, Y.; Deng, X.; Liang, J. Modulation of hepatic stellate cells and reversibility of hepatic fibrosis. Exp. Cell. Res. 2017, 352, 420-426. [CrossRef] [PubMed]

22. Liu, X.; Xu, J.; Brenner, D.A.; Kisseleva, T. Reversibility of liver fibrosis and inactivation of fibrogenic myofibroblasts. Curr. Pathobiol. Rep. 2013, 1, 209-214. [CrossRef] [PubMed]

23. Wasmuth, H.E.; Tacke, F.; Trautwein, C. Chemokines in liver inflammation and fibrosis. Semin. Liver Dis. 2010, 30, 215-225. [CrossRef] [PubMed]

24. Morrison, M.C.; Liang, W.; Mulder, P.; Verschuren, L.; Pieterman, E.; Toet, K.; Heeringa, P.; Wielinga, P.Y.; Kooistra, T.; Kleemann, R. Mirtoselect, an anthocyanin-rich bilberry extract, attenuates non-alcoholic steatohepatitis and associated fibrosis in ApoE*3Leiden mice. J. Hepatol. 2015, 62, 1180-1186. [CrossRef] [PubMed]

25. Singh, S.; Sadanandam, A.; Varney, M.L.; Nannuru, K.C.; Singh, R.K. Small interfering RNA-mediated CXCR1 or CXCR2 knock-down inhibits melanoma tumor growth and invasion. Int. J. Cancer 2010, 126, 328-336. [CrossRef] [PubMed]

26. Saintigny, P.; Massarelli, E.; Lin, S.; Ahn, Y.H.; Chen, Y.; Goswami, S.; Erez, B.; O’Reilly, M.S.; Liu, D.; Lee, J.J.; et al. CXCR2 expression in tumor cells is a poor prognostic factor and promotes invasion and metastasis in lung adenocarcinoma. Cancer Res. 2013, 73, 571-582. [CrossRef] [PubMed]

27. Matsuo, Y.; Raimondo, M.; Woodward, T.A.; Wallace, M.B.; Gill, K.R.; Tong, Z.; Burdick, M.D.; Yang, Z.; Strieter, R.M.; Hoffman, R.M.; et al. CXC-chemokine/CXCR2 biological axis promotes angiogenesis in vitro and in vivo in pancreatic cancer. Int. J. Cancer 2009, 125, 1027-1037. [CrossRef] [PubMed]

28. Nischalke, H.D.; Berger, C.; Lutz, P.; Langhans, B.; Wolter, F.; Eisenhardt, M.; Kramer, B.; Kokordelis, P.; Glassner, A.; Muller, T.; et al. Influence of the CXCL1 rs4074 A allele on alcohol induced cirrhosis and HCC in patients of European descent. PLoS ONE 2013, 8, e80848. [CrossRef] [PubMed] 
29. Chang, B.; Xu, M.J.; Zhou, Z.; Cai, Y.; Li, M.; Wang, W.; Feng, D.; Bertola, A.; Wang, H.; Kunos, G.; et al. Short- or long-term high-fat diet feeding plus acute ethanol binge synergistically induce acute liver injury in mice: An important role for CXCL1. Hepatology 2015, 62, 1070-1085. [CrossRef] [PubMed]

30. Bataller, R.; Brenner, D.A. Liver fibrosis. J. Clin. Investig. 2005, 115, 209-218. [CrossRef] [PubMed]

31. Forbes, S.J.; Russo, F.P.; Rey, V.; Burra, P.; Rugge, M.; Wright, N.A.; Alison, M.R. A significant proportion of myofibroblasts are of bone marrow origin in human liver fibrosis. Gastroenterology 2004, 126, 955-963. [CrossRef] [PubMed]

32. Troeger, J.S.; Mederacke, I.; Gwak, G.Y.; Dapito, D.H.; Mu, X.; Hsu, C.C.; Pradere, J.P.; Friedman, R.A.; Schwabe, R.F. Deactivation of hepatic stellate cells during liver fibrosis resolution in mice. Gastroenterology 2012, 143, 1073-83.e22. [CrossRef] [PubMed]

33. Pulli, B.; Ali, M.; Iwamoto, Y.; Zeller, M.W.; Schob, S.; Linnoila, J.J.; Chen, J.W. Myeloperoxidase-hepatocyte-stellate cell cross talk promotes hepatocyte injury and fibrosis in experimental nonalcoholic steatohepatitis. Antioxid. Redox Signal. 2015, 23, 1255-1269. [CrossRef] [PubMed]

34. Henderson, N.C.; Arnold, T.D.; Katamura, Y.; Giacomini, M.M.; Rodriguez, J.D.; McCarty, J.H.; Pellicoro, A.; Raschperger, E.; Betsholtz, C.; Ruminski, P.G.; et al. Targeting of alphav integrin identifies a core molecular pathway that regulates fibrosis in several organs. Nat. Med. 2013, 19, 1617-1624. [CrossRef] [PubMed]

35. Legate, K.R.; Wickstrom, S.A.; Fassler, R. Genetic and cell biological analysis of integrin outside-in signaling. Genes Dev. 2009, 23, 397-418. [CrossRef] [PubMed]

36. Li, Y.; Wu, J.; Song, F.; Tang, J.; Wang, S.J.; Yu, X.L.; Chen, Z.N.; Jiang, J.L. Extracellular membrane-proximal domain of HAb18G/CD147 binds to metal ion-dependent adhesion site (MIDAS) motif of integrin beta1 to modulate malignant properties of hepatoma cells. J. Biol. Chem. 2012, 287, 4759-4772. [CrossRef] [PubMed]

37. Li, Y.L.; Wu, J.; Wei, D.; Zhang, D.W.; Feng, H.; Chen, Z.N.; Bian, H. Newcastle disease virus represses the activation of human hepatic stellate cells and reverses the development of hepatic fibrosis in mice. Liver Int. 2009, 29, 593-602. [CrossRef] [PubMed]

38. Li, Y.; Xu, J.; Chen, L.; Zhong, W.D.; Zhang, Z.; Mi, L.; Zhang, Y.; Liao, C.G.; Bian, H.J.; Jiang, J.L.; et al. HAb18G (CD147), a cancer-associated biomarker and its role in cancer detection. Histopathology 2009, 54, 677-687. [CrossRef] [PubMed]

39. Wu, J.; Ru, N.Y.; Zhang, Y.; Li, Y.; Wei, D.; Ren, Z.; Huang, X.F.; Chen, Z.N.; Bian, H. HAb18G/CD147 promotes epithelial-mesenchymal transition through TGF-beta signaling and is transcriptionally regulated by Slug. Oncogene 2011, 30, 4410-4427. [CrossRef] [PubMed]

40. Wu, B.; Cui, J.; Yang, X.M.; Liu, Z.Y.; Song, F.; Li, L.; Jiang, J.L.; Chen, Z.N. Cytoplasmic fragment of CD147 generated by regulated intramembrane proteolysis contributes to HCC by promoting autophagy. Cell Death Dis. 2017, 8, e2925. [CrossRef] [PubMed] 